University of Nebraska - Lincoln

DigitalCommons@University of Nebraska - Lincoln

Steady-periodic heating in parallel-plate microchannel flow with participating walls

Kevin D. Cole

University of Nebraska-Lincoln, kcole1@unl.edu

Follow this and additional works at: https://digitalcommons.unl.edu/mechengfacpub

Part of the Mechanical Engineering Commons

Cole, Kevin D., "Steady-periodic heating in parallel-plate microchannel flow with participating walls" (2010). Mechanical \& Materials Engineering Faculty Publications. 55.

https://digitalcommons.unl.edu/mechengfacpub/55

This Article is brought to you for free and open access by the Mechanical \& Materials Engineering, Department of at DigitalCommons@University of Nebraska - Lincoln. It has been accepted for inclusion in Mechanical \& Materials Engineering Faculty Publications by an authorized administrator of DigitalCommons@University of Nebraska Lincoln. 
Published in International Journal of Heat and Mass Transfer 53:5-6 (February 2010), pp. 870-878;

doi:10.1016/j.ijheatmasstransfer.2009.11.037 Copyright ( 2009 Elsevier Ltd. Used by permission.

\title{
Steady-periodic heating in parallel-plate microchannel flow with participating walls
}

\author{
Kevin D. Cole \\ Mechanical Engineering Department, N104 Walter Scott Engineering Center, \\ University of Nebraska-Lincoln, Lincoln, NE 68588-0656, USA; email kcole1@unl.edu
}

\begin{abstract}
Simulations are presented for thermal sensing of steady laminar flow in a parallel-plate microchannel. Steady-periodic heating is introduced at the channel wall and temperatures are reported at upstream and downstream locations to represent temperature sensors. Exact analytical expressions for the temperature are given in the form of integrals, and numerical values are found by quadrature. Because axial conduction is prominent, there is a well-defined measurable flow range associated with each thermal-sensor geometry. Various fluid-flow rates, heating frequencies, sensor locations, and wall properties are explored. The results are given as a design correlation which shows the extent to which a given sensor can be tuned, by adjusting the heating frequency, to the flowmeasurement range of interest.
\end{abstract}

Keywords: axial conduction, thermal entrance, conjugate heat transfer, Green's function

\section{Introduction}

This paper is a theoretical study of periodic heating in a parallel-plate channel with participating walls. The application is measurement of fluid flow in microchannels for which axial conduction is important both in the fluid flow and in the channel walls. Pertinent literature will next be discussed in the areas of flows with axial conduction in the fluid, flows with thermally-participating walls, and flow-measurement applications in microchannels.

There have been several studies of thermal entrance problems in channel flows with fully-developed laminar velocity. The thermal entrance is characterized by an unheated wall for $x<0$ and a heated wall for $x>0$, and the heated region extends far down the channel. Both theoretical studies [1, 2] and numerical studies $[3,4]$ have identified the value of the Peclet number below which axial heat conduction in the fluid is important, and have studied the impact on the Nusselt number in entrance region. Several researchers included thermal participation of the channel walls which is strongest near the entrance region $[5,6]$ and can influence the overall performance of heat exchangers involving microchannel flow passages [7]. A heated region confined to a small region along the wall was studied by Weigand $[8,9]$, who found that a small heated region extends the impact of axial conduction to higher Peclet numbers. There have been several thermal-entrance studies of microchannels with other velocity distributions, including Hartmann flow [10, 11], porous-saturated flow [12], and slip flow [13]. Slip flow is important in a flowing gas when the mean-free-path $\lambda$ cannot be neglected compared to the channel height $L$. Specifically, if the Knudsen number, defined by $\lambda / L$, is in the range $(0.01,0.1)$, then slip flow is valid [14].
Most of the existing work on heat transfer in microchannels has been for steady heat transfer. However there has been some work on the entrance problem with time-varying inlet temperature [15, 16] which has application for thermal transients associated with heat exchangers.

There have been some thermal studies in microchannels for the purpose of flow measurement. Bedo et al. [17] carried out an experimental study of a heater flanked by two temperature sensors, one upstream and one downstream. The heating was steady-periodic and the amplitude and phase of the temperatures were measured. Use of steady-periodic heating for thermal sensing can improve the signal stability compared to DC signals, and, use of the phase of the signal can remove the need for temperature compensation of the signal. The upstream temperature signal gives good results for measurement of gas flow at low velocity. The downstream temperature is useful for water-flow measurement, but as flow increases, eventually the downstream temperature-flow curve saturates (the slope becomes zero). Bedo et al. suggest that the saturation point is determined by the fluid thermal properties. Yan et al. [18] used a similar sensor configuration (heater with two flanking temperature sensors), but instead used a steadily-energized heater and the sensing signal was the temperature difference between the downstream and upstream sensors. The flow calibration showed that as the flow increases, the signal is at first sensitive to flow speed, but that there is a point where the signal reaches a maximum and the flow sensitivity falls to zero. Thus, experimental studies indicate there is a clearly-defined flow range for which a particular sensor can be used.

In this paper simulations are given for the temperature in the parallel-plate microchannel caused by steady-periodic heating over a small region of the wall. An exact analyt- 


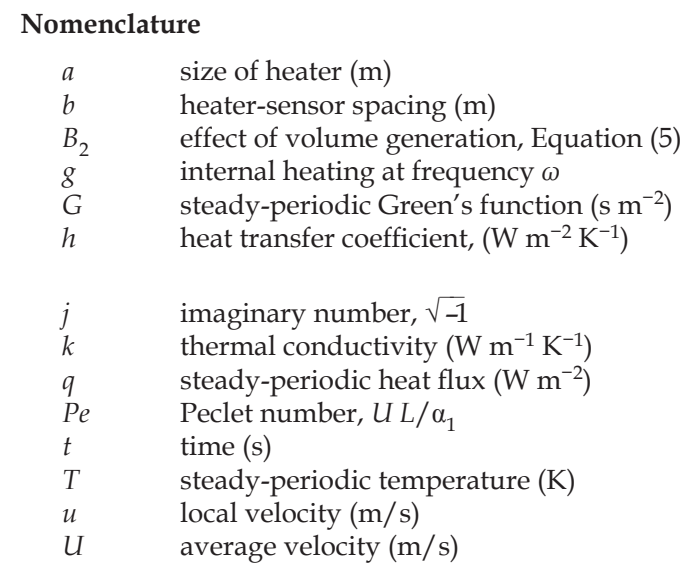

ical solution, based on the method of Green's functions, gives the temperature in the form of integrals, and numerical values are computed with quadrature. Axial conduction in the fluid and in the adjacent wall are included in the theory. The velocity distribution in the channel is fully-developed laminar, appropriate for liquid flow or for gas flow with sufficiently small Knudsen number. The contributions of this paper are: firstly, to present simulations of thermal sensors applied to flow measurement in microchannels; secondly, to explain the previously-observed maximum-signal phenomenon that limits the useful flow-observation range of such sensors; and thirdly, to present correlations that predict the useful range of flow values that can be observed with thermal sensors, for the purpose of sensor design. The paper is divided into sections on the temperature equations, the Green's function solution, numerical results, and conclusions.

\section{Temperature equations}

The equations describing the temperature in the parallelplate flow and in the adjacent wall are given in this section. The geometry is shown in Figure 1. Symmetrically- placed heaters are located at the surface of a fully-developed laminar flow between the plates. The plate spacing is $L$ and the wall thickness is $W$. Since the discussion is limited to steady-periodic heating, we take the introduced heat and the resulting temperature to be steady periodic at a single frequency. The temperature satisfies the following equations:

$$
\begin{aligned}
& \frac{\partial^{2} T_{1}}{\partial x^{2}}+\frac{\partial^{2} T_{1}}{\partial y_{1}^{2}}=\frac{u\left(y_{1}\right)}{\alpha_{1}} \frac{\partial T_{1}}{\partial x}+\frac{j \omega}{\alpha_{1}} T_{1} \\
& \frac{\partial^{2} T_{2}}{\partial x^{2}}+\frac{\partial^{2} T_{2}}{\partial y_{2}^{2}}=-\frac{1}{k_{2}} g\left(x, y_{2}, \omega\right)+\frac{j \omega}{\alpha_{2}} T_{2} \\
& \left.k \frac{\partial T_{2}}{\partial y_{2}}\right|_{y_{2}=W}+\left.h T_{2}\right|_{y_{2}=W}=f(x, \omega) ;\left.\quad \frac{\partial T_{1}}{\partial y_{1}}\right|_{y_{1}=L / 2}=0
\end{aligned}
$$

In this paper complex-valued $T_{i}(x, y, \omega)$ is interpreted as the steady-periodic temperature (Kelvin) in the $i$ th body ( $i=1$ or 2 ) at a single frequency $\omega$. For further discussion of this point see [19, pp. 2-3]. Later in the paper, results will be discussed in the form of amplitude and phase of this temperature.

The non-fluid side of the channel wall is described by a general-purpose boundary condition of the third kind (con-

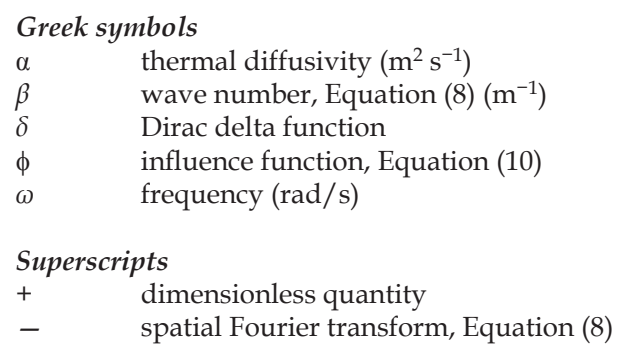

$\begin{array}{ll}\text { Subscripts } \\ 1 & \text { fluid } \\ 2 & \text { solid } \\ h & \text { heater }\end{array}$

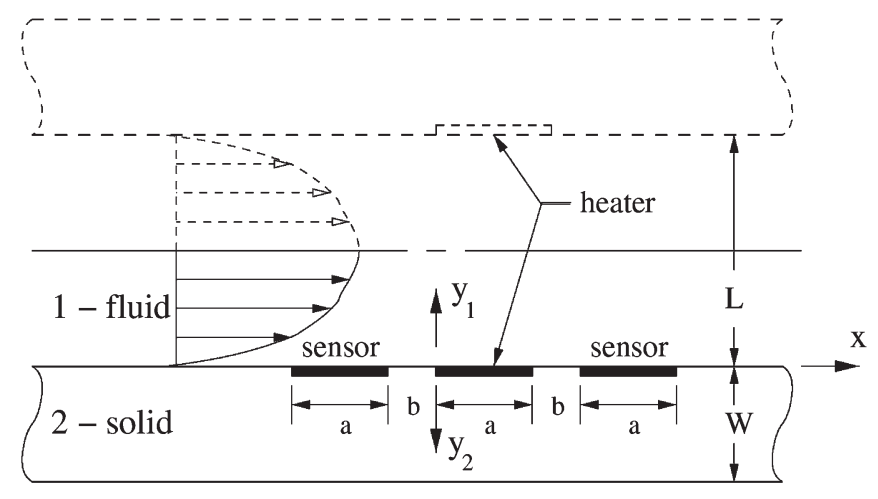

Figure 1. Geometry of flat-plate channel flow with symmetric heaters and with upstream and downstream temperature sensors.

vection); this condition can also be used to provide a specified temperature condition (first kind) for $h \rightarrow \infty$, and the specified flux condition (second kind) for $h=0$. Later numerical results are presented for the insulated condition (flux specified to be zero), to represent a repeating pattern of equally-spaced flow channels separated by walls of thickness $2 W$.

\section{GF solution}

The steady-periodic temperature is developed with the Green's function (GF) method combined with a spatial Fourier transform along the $x$-direction. As this approach was used in an earlier paper describing external flow [20], only a brief outline of the solution procedure is given here.

Let region 2 be a stationary solid heated by a known volume energy source, $g(x, y, \omega)$, and let region 1 be a flowing fluid which is heated by contact with region 2 . Then the temperatures in each region may be formally stated with the method of GF in terms of known GF named $G_{1}$ and $G_{2}$ and unknown interface heat fluxes $q_{1}$ and $q_{2}$, as follows [21, Ch. 3]:

$$
\begin{aligned}
& T_{1}\left(x, y_{1}, \omega\right)=\frac{\alpha_{1}}{k_{1}} \int q_{1}\left(x^{\prime}, \omega\right) G_{1}\left(x-x^{\prime}, y_{1}, y_{1}^{\prime}=0, \omega\right) d x^{\prime} \\
& T_{2}\left(x, y_{2}, \omega\right)=\frac{\alpha_{2}}{k_{2}} \int q_{2}\left(x^{\prime}, \omega\right) G_{2}\left(x-x^{\prime}, y_{2}, y_{2}^{\prime}=0, \omega\right) d x^{\prime}+B_{2}\left(y_{2}\right)
\end{aligned}
$$


where

$$
B_{2}\left(x, y_{2}, \omega\right)=\frac{\alpha_{2}}{k_{2}} \iint g\left(x^{\prime}, y_{2}^{\prime}, \omega\right) G_{2}\left(x-x^{\prime}, y_{2}, y_{2}^{\prime}\right) d x^{\prime} d y_{2}^{\prime}
$$

Here $B_{2}$ is the contribution to the temperature caused by the volume energy source, $g$. In the equations given above, the boundary heat fluxes and the boundary temperatures are unknown, but they are related by matching conditions at the interface between the regions. The heat flux entering region 1 leaves region 2 , and the temperatures match at the interface. That is,

$$
\begin{aligned}
& q_{1}(x, \omega)=-q_{2}(x, \omega) \\
& T_{1}(x, 0, \omega)=T_{2}(x, 0, \omega)
\end{aligned}
$$

Next the Fourier transform will be used to strip away the integrals and allow for an algebraic solution in Fourier-transform space. The Fourier transform is defined by the following transform pair:

$$
\begin{aligned}
& \bar{T}(\beta)=\int_{-\infty}^{\infty} T(x) e^{-j \beta x} d x \\
& T(x)=\frac{1}{2 \pi} \int_{-\infty}^{\infty} \bar{T}(\beta) e^{j \beta x} d \beta
\end{aligned}
$$

Apply the integral transform to Equations (4)-(7), evaluate all the temperatures at the fluid-solid interface, carry out an algebraic solution for the unknown heat flux to the fluid $\bar{q}_{1}(\beta)$, and then substitute the heat flux back into the temperature expression in the fluid, Equation (4). Then the solution for the fluid temperature in Fourier space is given by

$$
\bar{T}_{1}\left(\beta, y_{1}=0, \omega\right)=\frac{\bar{B}_{2}}{\phi_{2}+\phi_{1}} \phi_{1}
$$

Here influence function $\phi_{i}$ is the Fourier-space GF multiplied by a ratio of thermal properties in the form $\phi_{i}=\left(\alpha_{i} / k_{i}\right) \bar{G}_{i}$. Finally, the temperature in real space is found with the inverse Fourier transform,

$$
T_{1}\left(x, y_{1}=0, \omega\right)=\frac{1}{2 \pi} \int_{-\infty}^{\infty}\left[\frac{\bar{B}_{2}}{\phi_{2}+\phi_{1}} \phi_{1}\right] e^{j \beta x} d \beta
$$

The two-region solution given here is appropriate for the symmetrically-heated channel. A non-symmetrically heated channel could have been treated by a three-region solution (heated wall, fluid, unheated wall) again with an exact algebraic solution in Fourier space. The GF method can be used for any number of regions in contact (see for example [22]). The above temperature expression applies to any heating distribution in the wall. Next, the specific form of the heating function $\bar{B}_{2}$ will be introduced for a thin heater located at the fluid-solid interface.

\subsection{Thin, flush-mounted heater}

In the special case of a thin, flush-mounted heater, the volume generation term takes the form $g\left(x, y_{2}, \omega\right)=q_{h}(x, \omega) \delta\left(y_{2}\right)$ where $q_{h}$ is the heater flux and $\delta$ is the Dirac delta function. Now replace this form of $g$ into the expression for $B_{2}$, evaluate the integral over $y_{2}$ with the sifting property of the Dirac delta function, and strip off the integral over $x^{\prime}$ with the spatial-Fourier transform:

$$
\bar{B}_{2}\left(\beta, y_{2}, \omega\right)=\frac{\alpha_{2}}{k_{2}} \bar{q}_{h}(\beta, \omega) \bar{G}_{2}\left(\beta, y_{2}, \omega\right)=\bar{q}_{h} \phi_{2}
$$

Then the interface temperature is given by

$$
T_{1}\left(x, y_{1}=0, \omega\right)=\frac{1}{2 \pi} \int_{-\infty}^{\infty}\left[\bar{q}_{h} \frac{\phi_{2} \phi_{1}}{\phi_{2}+\phi_{1}}\right] e^{j \beta x} d \beta
$$

For the special case of spatially-uniform heat-flux introduced by the heater, quantity $q_{h}$ in $x$-space is given by

$$
q_{h}(x, \omega)= \begin{cases}q_{0} ; & 0<x<a \\ 0 ; & \text { otherwise }\end{cases}
$$

where $q_{0}$ is a constant. Substitute the spatial-Fourier transform of this heat flux into the above temperature expression, to find

$$
T_{1}\left(x, y_{1}=0, \omega\right)=\frac{1}{2 \pi} \int_{-\infty}^{\infty}\left[q_{0} \frac{1-e^{-j \beta a}}{j \beta} \frac{\phi_{1} \phi_{2}}{\phi_{1}+\phi_{2}}\right] e^{j \beta x} d \beta
$$

This is the temperature at the fluid-solid interface $\left(y_{1}=0\right)$ caused by a thin, uniform heater located on $(0<x<a)$.

In the present work a thin flush-mounted heater is used for simplicity, and for comparison with previous work. A thick heater could be accommodated by including a spatially-distributed heating function $g$ within heating integral $\bar{B}_{2}$, with the approximation that the heater thermal properties are close to those of the wall. A more precise simulation of a thick heater (or thick sensor) would involve a three-region solution (fluid, heater/sensor, wall). See for example [23].

\subsection{Spatial average on the interface}

The above temperature expression for the temperature at a point is important for understanding the thermal mechanisms present in microchannel flow. However for flow-measurement applications, it is the average temperature over a region on the interface that is more relevant, in order to simulate a finitesized thermal sensor.

Two types of thermal sensors will be discussed here. First, a thin metal film can serve both as heater and temperature sensor if suitable electronics are employed such as those developed for pulse-heated thermal conductivity measurements [24]. Second, additional thin sensors can be placed on the fluid-solid interface, such as a metal film with electrical resistance calibrated for temperature.

Of interest is the spatial average temperature on a region $(\xi<x<\xi+a)$ of the interface. This average temperature may be computed from the surface temperature by

$$
\begin{aligned}
T_{a v}(\xi, \omega) & =\frac{1}{a} \int_{\xi}^{\xi+a} T_{1}(x, 0, \omega) d x \\
& =\frac{1}{a} \int_{\xi}^{\xi+a}\left[\frac{1}{2 \pi} \int_{-\infty}^{\infty} \bar{T}_{1}(\beta, 0, \omega) e^{j \beta x} d \beta\right] d x \\
& =\frac{1}{2 \pi} \int_{-\infty}^{\infty} \bar{T}_{1}(\beta, 0, \omega)\left[\frac{e^{j \beta \xi}\left(e^{j \beta a}-1\right)}{j \beta a}\right] d \beta .
\end{aligned}
$$

Here the spatial integral over $x$ has been carried out in closed form. Quantity $T_{a v}$ is actually easier to evaluate numerically, compared to $T_{1}$, because of the introduction of factor $1 / \beta$ which causes the integrand to vanish more rapidly as $\beta \rightarrow \pm \infty$. Average temperatures computed from the above expression will be reported later in this paper for the heater and for sensors located upstream and downstream of the heater.

An advantage of the GF method is that the same solution procedure applies for any fluid velocity distribution and for any wall-side geometry as long as the appropriate GF are used. The solid-side GF has been discussed previously [20]; in the next section the fluid-side GF is developed.

\section{GF for flow between parallel plates}

In this section the Green's function is developed for the steady-periodic temperature in a fully-developed flow between parallel plates. The Green's function associated with the 
time-periodic problem in the fluid, Equation (1), satisfies the following energy equation:

$$
\frac{\partial^{2} G_{1}}{\partial x^{2}}+\frac{\partial^{2} G_{1}}{\partial y^{2}}=\frac{u(y)}{\alpha} \frac{\partial G_{1}}{\partial x}+\frac{j \omega}{\alpha} G_{1}-\frac{1}{\alpha} \delta\left(x-x^{\prime}\right) \delta\left(y-y^{\prime}\right)
$$

Note that in this section coordinate $y_{1}$ has been replaced with $y$ and $\alpha_{1}$ has been replaced by $\alpha$ to streamline the notation. The boundary conditions are

$$
\begin{aligned}
& \text { At } y=0, \quad \frac{\partial G_{1}}{\partial y}=0 \\
& \text { at } y=L / 2, \quad \frac{\partial G_{1}}{\partial y}=0 \\
& \text { at } x \rightarrow \pm \infty, \quad \partial G_{1} \text { is bounded. }
\end{aligned}
$$

A type 2 boundary (specified flux) is used at $y=0$ to be consistent with the flux condition used in Equation (6). For fully developed laminar flow between parallel plates, the fluid velocity distribution is given by

$$
u(y)=6 U\left[\frac{y}{L}-\left(\frac{y}{L}\right)^{2}\right]
$$

The solution for $G_{1}$ will be found with a Fourier transform along $x$. First, use a simple change of variable to replace $\left(x-x^{\prime}\right)$ by $x$. Then apply the Fourier transform, Equation (8), to the above differential equation to find

$$
\frac{d^{2} \bar{G}_{1}}{\partial y^{2}}-\left(\beta^{2}+\frac{j \omega}{\alpha}+6 U\left[\frac{y}{L}-\left(\frac{y}{L}\right)^{2}\right] \frac{j \beta}{\alpha}\right) \bar{G}_{1}=-\frac{1}{\alpha} \delta\left(y-y^{\prime}\right) .
$$

The next step is to find two independent solutions of the homogeneous differential equation, here named $\eta(y)$ and $\zeta(y)$. Consider first function $\eta(y)$ which satisfies the homogeneous version of Equation (22), given by

$$
\frac{d^{2} \eta}{\partial(y / L)^{2}}-\left[A+B \frac{y}{L}-B\left(\frac{y}{L}\right)^{2}\right] \eta=0
$$

where

$$
A=(\beta L)^{2}+j \omega L^{2} / \alpha ; \quad B=6 j(U L / \alpha) \beta L
$$

Note that Equation (23) has been multiplied by $L^{2}$ to make quantities $A$ and $B$ dimensionless. Function $\eta$ has the form of a hypergeometric series [6],

$$
\eta(y)=\sum_{m=0}^{\infty} C_{m}\left(\frac{y}{L}\right)^{m}
$$

where coefficients $C_{m}$ must be determined. Replace this series into the above differential equation, Equation (23), to find

$$
\begin{aligned}
& \sum_{m=2}^{\infty} m(m-1) C_{m}\left(\frac{y}{L}\right)^{m-2}-A \sum_{m=0}^{\infty} C_{m}\left(\frac{y}{L}\right)^{m}-B \sum_{m=0}^{\infty} C_{m}\left(\frac{y}{L}\right)^{m+1} \\
& \quad+B \sum_{m=0}^{\infty} C_{m}\left(\frac{y}{L}\right)^{m+2}=0
\end{aligned}
$$

Because polynomials are linearly independent, the above equation will be satisfied only if the coefficients sum to zero for each power of $y$. Beginning with power zero,

For $y^{0}: 2 \cdot 1 \cdot C_{2}-A C_{0}=0 \rightarrow C_{2}=A C_{0} / 2$

For $y^{1}: 3 \cdot 2 \cdot C_{3}-A C_{1}-B C_{0}=0 \rightarrow C_{3}=\left(A C_{1}+B C_{0}\right) / 6$

For $y^{2}: 4 \cdot 3 \cdot C_{4}-A C_{2}-B C_{1}+B C_{0}=0 \rightarrow C_{4}=\left(A C_{2}+B C_{1}-B C_{0}\right) / 12$

For $y^{m-2}: \quad C_{m}=\frac{1}{m(m-1)}\left[A C_{m-2}+B C_{m-3}-B C_{m-4}\right]$
In this way a recursion relation has been developed for coefficient $C_{m}$. The recursion relation alone is not sufficient to determine all the coefficients, as $C_{0}$ and $C_{1}$ may be chosen arbitrarily. In this work function $\eta(y)$ is chosen with $C_{0}=0$ and $C_{1}=1$. The other solution, $\zeta(y)$, is developed in the same fashion, with the same recursion relation, however the coefficients for $\zeta(y)$ are chosen to begin $C_{0}=1$ and $C_{1}=0$ to insure that $\eta(y)$ and $\zeta(y)$ are linearly independent. In summary, solutions $\eta(y)$ and $\zeta(y)$ are given by

$$
\begin{aligned}
& \eta(y)=0+1 \cdot \frac{y}{L}+0+\frac{A}{6}\left(\frac{y}{L}\right)^{3}+\frac{B}{12}\left(\frac{y}{L}\right)^{4}+\cdots \\
& \zeta(y)=1+0+\frac{A}{2}\left(\frac{y}{L}\right)^{2}+\frac{B}{6}\left(\frac{y}{L}\right)^{3}+\left(\frac{A^{2}}{24}-\frac{B}{12}\right)\left(\frac{y}{L}\right)^{4}+\cdots
\end{aligned}
$$

and where higher order terms are given by the recursion relation, Equation (27).

The convergence of the series for $\eta(y)$ and $\zeta(y)$ is controlled by coefficients $C_{m}$. On the plus side is that $C_{m}$ is divided by factor $m(m-1)$ which combined with recursion suggests that $C_{m}$ will shrink rapidly as $m$ increases. On the minus side, however, coefficient $C_{m}$ is multiplied by powers of the form ( $A$ and/or $B)^{m-2}$ which tend to grow as $m$ increases. Thus, the size of parameter $A$ (and parameter $B$ ) may be a factor in determining the convergence speed of the series. Parameters $A$ and $B$, defined in Equation (24), contain parameters for frequency $\omega$, flow rate $\bar{U}$, and wave number squared $\beta^{2}$. This suggests that convergence of the hypergeometric series may be a challenge for large frequencies, large flow rates, and large wave numbers.

Now that two independent homogeneous solutions $\eta(y)$ and $\zeta(y)$ are available, the Green's function that satisfies Equation (22) will be sought in the form

$$
\bar{G}_{1}\left(y, y^{\prime}\right)= \begin{cases}D_{1} \eta(y)+D_{2} \zeta(y) ; & y<y^{\prime} \\ D_{3} \eta(y)+D_{4} \zeta(y) ; & y>y^{\prime}\end{cases}
$$

Coefficients $D_{1}, D_{2}, D_{3}$, and $D_{4}$ are determined from the two boundary conditions at $y=0$ and $y=L / 2$ and from two matching conditions at $y=y^{\prime}$ (see for example [25, Ch. 1]). After considerable algebra, the Green's function is given by

$\bar{G}_{1}\left(y, y^{\prime}\right)=\frac{L}{\alpha} \begin{cases}{\left[-\eta\left(y^{\prime}\right) \zeta^{\prime}(L / 2)+\zeta^{\prime}(L / 2) \eta\left(y^{\prime}\right)\right] \zeta(y) / E ;} & y<y^{\prime} \\ {\left[-\eta(y) \zeta^{\prime}(L / 2) \zeta\left(y^{\prime}\right)+\eta^{\prime}(L / 2) \zeta\left(y^{\prime}\right) \zeta(y)\right] / E ;} & y>y^{\prime}\end{cases}$

where $E=\zeta^{\prime}(L / 2)\left[\eta^{\prime}\left(y^{\prime}\right) \zeta\left(y^{\prime}\right)-\zeta^{\prime}\left(y^{\prime}\right) \eta\left(y^{\prime}\right)\right]$

Here $\eta^{\prime}=\partial \eta / \partial(y / L)$ and $\zeta^{\prime}=\partial \zeta / \partial(y / L)$. For the special case of heating on the $y^{\prime}=0$ wall, the above expression may be simplified with the following relations:

$$
\begin{array}{ll}
\eta(0)=0 ; & \eta^{\prime}(0)=1 \\
\zeta(0)=1 ; & \zeta^{\prime}(0)=0
\end{array}
$$

Then

$$
\bar{G}_{1}(y, 0)=\frac{L}{\alpha}\left[-\eta(y)+\frac{\eta^{\prime}(L / 2)}{\zeta^{\prime}(L / 2)} \zeta(y)\right]
$$

This is the form of the fluid-side GF used for the numerical calculations presented in this paper.

\section{Temperature in fluid flow alone}

The response of the fluid alone, without wall effects, is discussed in this section. When the fluid dominates the heat transfer, this is equivalent to $k_{2} / k_{1} \rightarrow 0$ which causes $\phi_{2} \rightarrow \infty$. Then the solid influence function cancels out of Equation (13) to give the temperature in the fluid alone: 


$$
T_{1}\left(x, y_{1}=0, \omega\right)=\frac{1}{2 \pi} \int_{-\infty}^{\infty} \bar{q}_{h} \phi_{1} e^{j \beta x} d \beta
$$

\subsection{Agreement with limiting cases}

The thermal response of the fully-developed laminar flow in a channel has been checked against that for shear-flow velocity published previously [20]. Figure 2 is a comparison between the amplitude of the average heater temperature for both the quadratic velocity and shear-flow velocity at $a / L=0.5$ and for three heating frequencies. As the Peclet number increases, the temperatures for the different velocity distributions converge. The reason is that for sufficiently high flow rate in the channel, the thermal boundary layer introduced by the heater will be thin, and contained entirely within the linear portion of the laminar (quadratic) velocity distribution. So, the temperature response should tend towards that predicted by the shear flow theory.

At higher frequency there is closer agreement between the quadratic velocity and the shear-flow velocity. As frequency increases, the thermal penetration caused by the periodic heating will decrease in size, and eventually the thermal layer will be confined to the linear portion of the quadratic velocity distribution. One reason the temperature for the quadratic velocity is higher at low frequency is that quadratic theory is constrained at $y=L$ by an insulated channel boundary. The linear-velocity theory, taken from an external-flow study, is unbounded along $y$.

Another limiting case, when the Peclet number is very low, is simple conduction heat transfer. At low Peclet number, axial conduction moves heat more readily than bulk fluid motion. This limiting case is visible in Figure 2 where the temperature curves become flat at low Peclet number. That is, the temperature no longer depends on fluid motion. The quadratic-velocity theory agrees with two-dimensional conduction theory [26] within five decimal places for $P e=0.01$. The agreement with limiting cases described here provides very strong evidence that the quadratic-velocity theory is correct.

\subsection{Heater temperature}

Previous studies have shown that a flush-mounted metal film can be used both to introduce heat and to provide a temperature signal that is useful for measurement of external flow [20]. In this section the combined heater/sensor will be discussed.

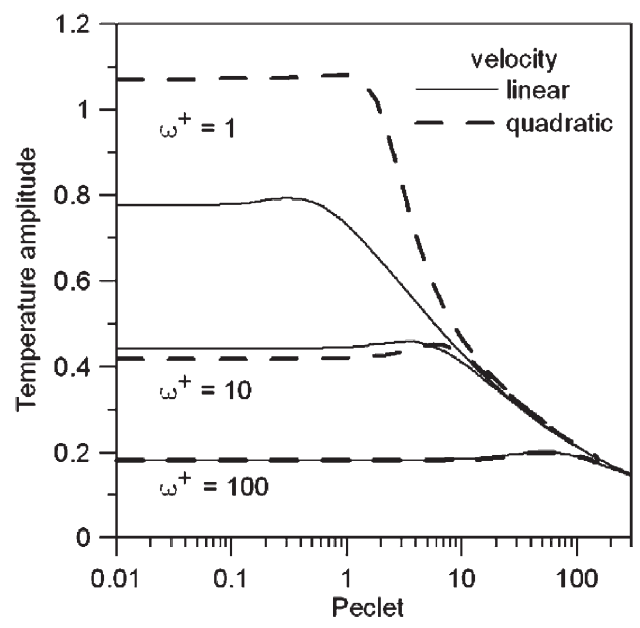

Figure 2. Heater temperature comparison between shear flow (linear) velocity and channel (quadratic) velocity, for three dimensionless frequencies.

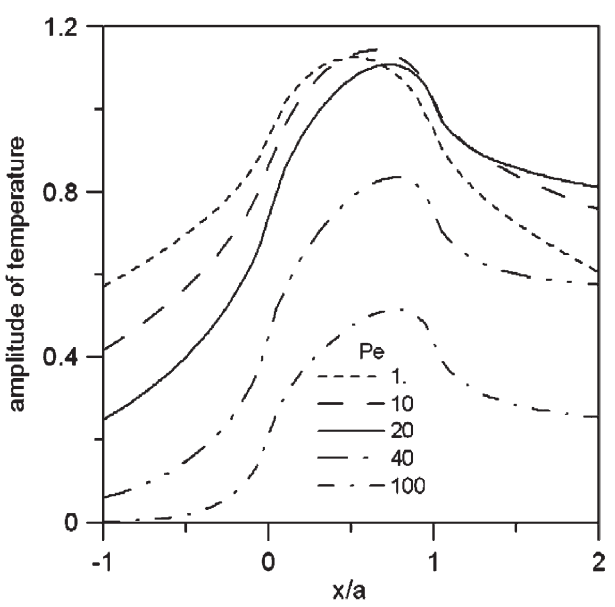

Figure 3. Temperature amplitude along the wall in channel flow for heating over region $(0<x / a<1)$, for frequency $\omega^{+}=1$, and for several flow values. Flow is from left to right, and no wall effects are included.

Figure 3 shows the temperature amplitude plotted versus location along the wall. The heater is located over the region $(0<x / a<1)$. The flow is from left to right, and the heating frequency is $\omega^{+}=\omega L^{2} / \alpha_{1}=1$. At low flow rates the temperature distribution is symmetric upstream and downstream, caused by axial conduction in the fluid. As the fluid flow increases, the temperature on the upstream edge of the heater decreases monotonically, cooled by the increasing convection heat transfer. However, on the downstream edge of the heater, the temperature first increases somewhat with fluid flow (as convection begins to rearrange heat from upstream), then reaches a maximum (when convection and conduction are about equal) and then decreases as the flow speed increases further (when convection heat transfer is dominant). It is important to note that the reversing trend in temperature amplitude is caused by axial conduction in the fluid. This trend is not limited to microchannel flows, as it appears in the shear-flow results shown in Figure 2 as a small upward hump. In external flow this upward hump is more prominent at higher frequencies, as a percentage of maximum amplitude. The prominence at higher frequencies occurs because the thinner thermal boundary layer resides in a lower-velocity region in which axial conduction may play a role. This effect was observed in earlier work by the author on external flow [20] but was not fully understood at that time.

This upward hump in temperature amplitude will next be discussed for microchannel flow. The spatial average temperature on the heater, the simulated sensor signal, is shown in Figure 4. Consider first the top curve in Figure 4a, the amplitude of heater temperature, plotted versus $P e$ at $\omega^{+}=10$. At very low fluid flow, there is no sensitivity to fluid flow (the curve is flat). Next as the flow (Peclet number) increases, the amplitude first rises, then falls. This is a confounding effect for flow measurement. That is, in the range of this amplitude reversal, a measured temperature amplitude could be caused by either one of two values of the flow. The combined heater/sensor, then, could only be used if the flow speed at which the peak temperature occurs has been identified ahead of time. This peak temperature must be avoided, because at that point the flow-sensitivity of the temperature amplitude is zero (zero slope).

The phase of the heater temperature, shown in Figure $4 b$, does not reverse, but changes monotonically with the flow. This suggests that phase is better than amplitude for flow measurement. As the $P e$ value increases further, the phase curve 

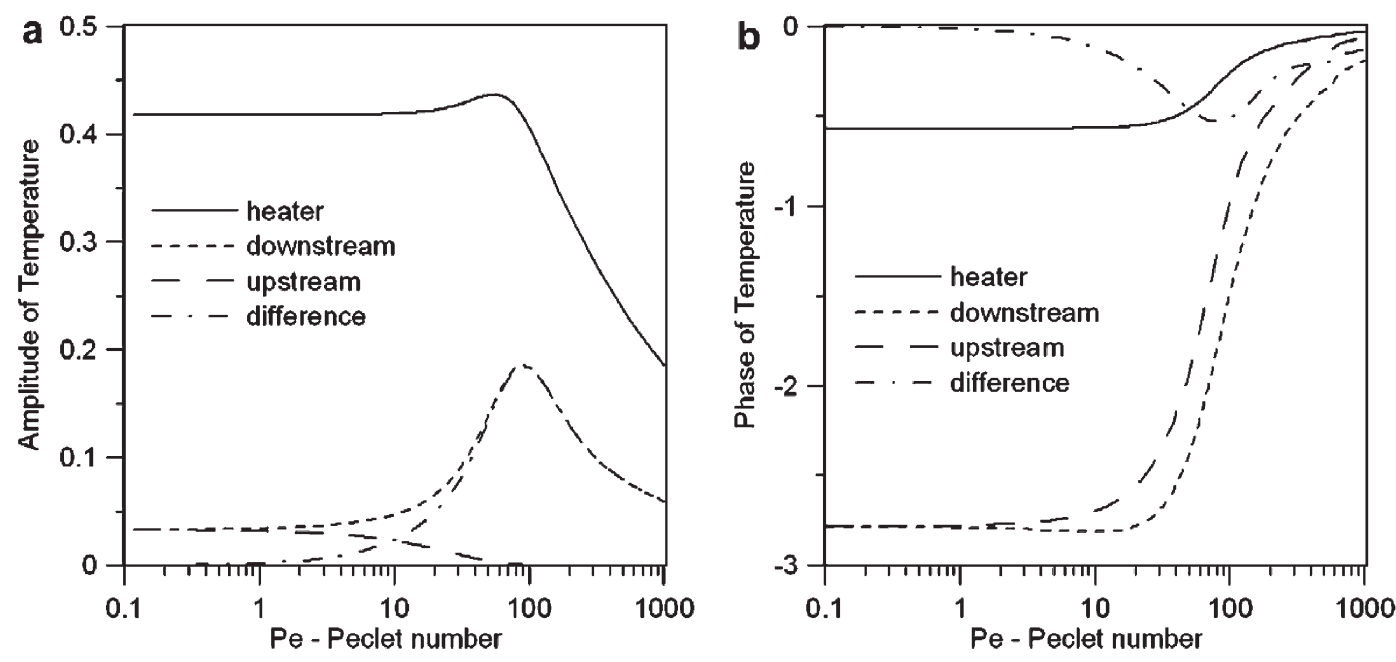

Figure 4. Temperature amplitude and phase for heater of size $a / L=0.5$ and for upstream and downstream sensors at distance $b / a=1$. The heating frequency is $\omega^{+}=10$. Heat transfer in fluid only.

flattens out, signifying that there is a specific range for which the flow sensitivity is good (large slope) and beyond this range the sensitivity of phase to the fluid flow tends to zero (in this case $P e>400$ ). This behavior was described by Bedo et al. [17] as saturation of the sensor at large flow.

\subsection{Upstream and downstream temperature sensors}

Next the temperatures from the upstream and downstream sensors, also shown in Figure 4, will be discussed. At small Pe values, the upstream and downstream temperatures in Figure 4 start out at the same value, where conduction dominates. As $P e$ increases, these temperatures begin to respond to flow earlier than the heater temperature, at about $P e \approx 1$. As $P e$ increases further the upstream temperature falls monotonically as discussed earlier, and the downstream temperature first rises, reaches a peak, and then falls after convection begins to dominate the heat transfer there. Note that the overall temperature change for the downstream sensor is greater than that for the heater temperature.

The temperature difference between the downstream sensor and the upstream sensor is also plotted in Figure 4. This temperature difference has a special appeal for flow measurement, because both phase and amplitude values start from zero. No DC component needs to be subtracted from the signal. As $P e$ increases the temperature difference signal shows a shape similar to the downstream temperature alone, however the overall signal change is greatest of all. That is, the difference signal gives the greatest flow sensitivity, and it responds to smaller flow values than the heater temperature alone. Measurement of low-flow values is particularly important for the microchannel application, which suggests that the difference signal is more advantageous than the heater temperature.

\section{Temperature with participating walls}

Thermal participation of the walls is important when the wall has thermal conductivity greater than or equal to that of the fluid. The base case discussed here is water flow through a glass-walled channel, see Table 1 for the thermal properties used here. Figure $5 \mathrm{a}$ and $\mathrm{b}$ show the amplitude and phase, respectively, of the temperature on the heater and sensors plotted versus Peclet number at frequency $\omega^{+}=10$. Consider first
Table 1. Thermal property ratios used in simulations. Values for water are $k_{1}=0.611 \mathrm{~W} / \mathrm{m} / \mathrm{K}$ and $\alpha_{1}=0.147\left(10^{-6}\right) \mathrm{m}^{2} / \mathrm{s}$.

\begin{tabular}{lccll}
\hline Material & $\mathrm{k}_{2}\left(\frac{\mathrm{W}}{\mathrm{mK}}\right)$ & $\alpha_{2}\left(\frac{\mathrm{m}^{2}}{\mathrm{~s}}\right)$ & $\frac{k_{1}}{k_{2}}$ & $\frac{\alpha_{1}}{\alpha_{2}}$ \\
\hline Plastic & 0.23 & $0.10\left(10^{-6}\right)$ & 2.66 & 1.47 \\
Glass & 1.38 & $0.83\left(10^{-6}\right)$ & 0.443 & 0.177 \\
Stainless steel & 13. & $3.40\left(10^{-6}\right)$ & 0.047 & 0.0432 \\
\hline
\end{tabular}

the heater temperature, which is insensitive to the flow until $P e>20$. The shape of the heater amplitude curve is nearly unchanged compared to the fluid flow alone, however the amplitude is somewhat lower when the wall is participating (see Figure 4). In addition, the range of phase values is smaller when the wall is participating. That is, the presence of the wall decreases the flow sensitivity of the sensor, whether amplitude or phase is used for measurement.

The discussion so far has shown that there is a flow value at which the sensor signal peaks in amplitude and flattens out in phase. This flow value represents the upper limit of the measurable flow range. Using the thermal simulation, several frequencies were explored to see the effect of frequency on the useful flow range. The peak amplitude in the temperature-difference signal (downstream minus upstream) was chosen as a measure of the maximum measureable Peclet number. Although it is true that the phase of the difference signal keeps changing with flow above the peak amplitude (see Figure 5b), the phase slope (sensitivity) is falling in this range, which indicates that the practical upper limit of Peclet number is not far away. So, the use of the peak amplitude, while not the ultimate maximum, is an unambiguous marker for the maximum observable Peclet value.

Figure 6 shows the temperature difference signal plotted versus Peclet number for several frequency values for sensor geometry $b / a=1.0$ and $a / L=0.5$. Both amplitude and phase are given. The figure shows that as frequency increases the peak shifts to higher Peclet values. Thus a fixed-geometry sensor can be tuned, by changing the frequency, to the flow range of interest. The range of phase values increases as the frequency increases, suggesting that a phase-based measurement will have higher resolution at higher frequency. There is a tradeoff, however, as the signal amplitude decreases as frequency increases. That is, high frequency signals will be more difficult to detect compared to low frequency signals. 

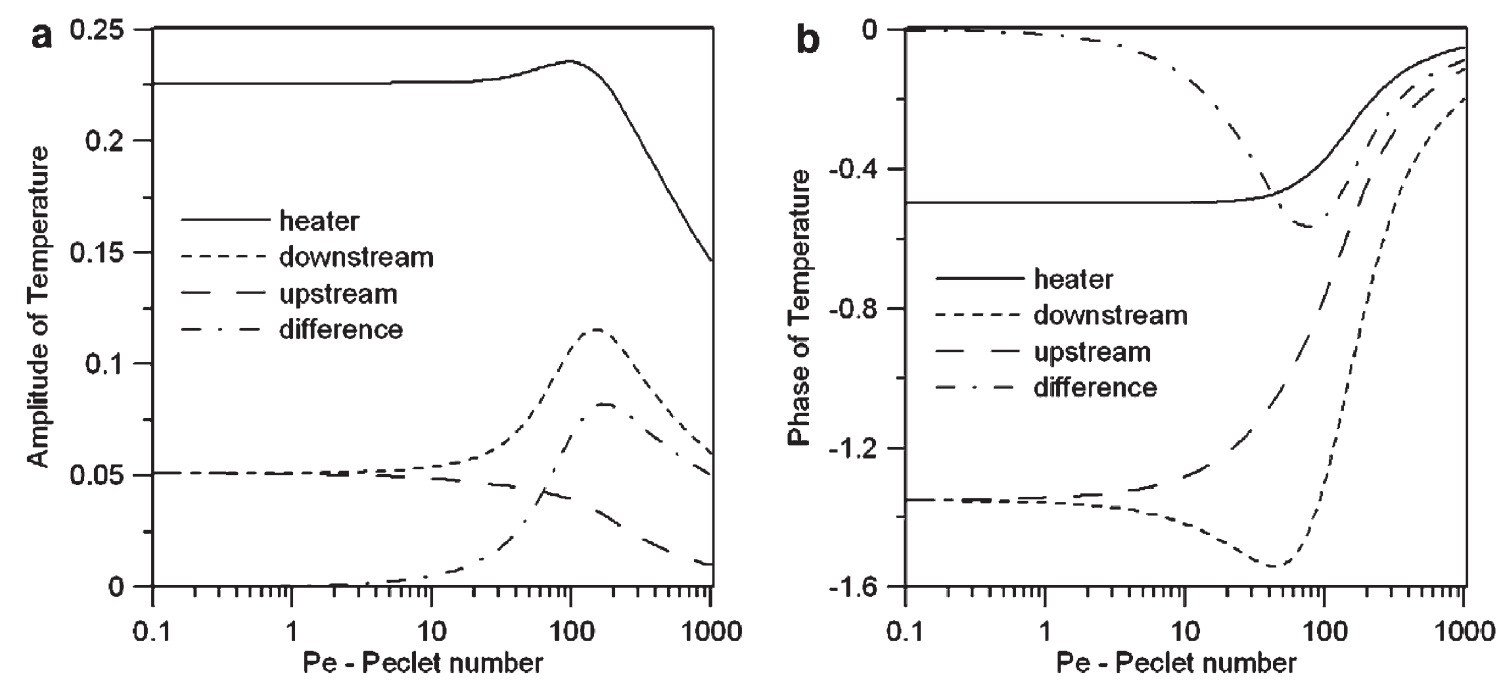

Figure 5. Temperature amplitude and phase for heating at frequency $\omega^{+}=10$ for water flow in a glass-walled microchannel $\left(k_{1} / k_{2}=0.443\right)$. The heater size is $a / L=0.5$, the heater-sensor spacing is $b / a=1$, and the wall thickness is $W / L=0.5$.
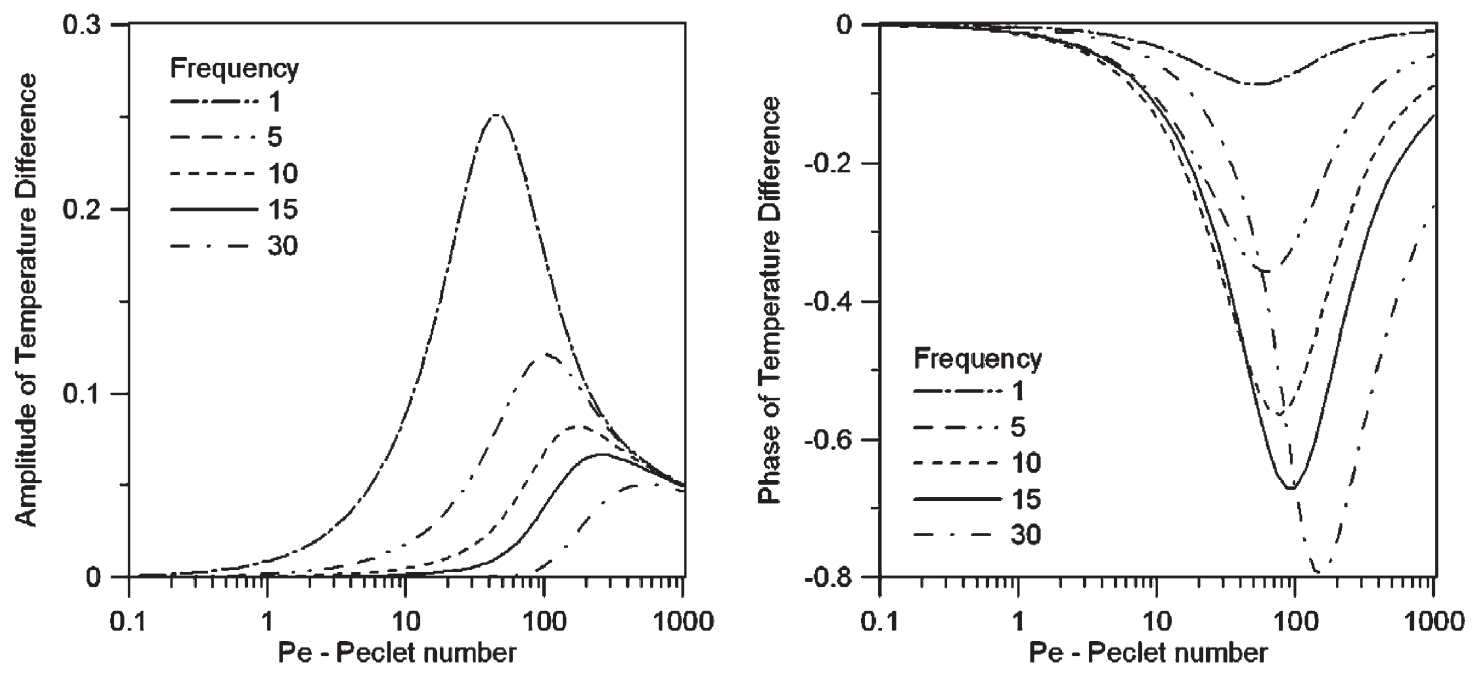

Figure 6. Amplitude and phase of the temperature difference (downstream minus upstream) for several heating frequencies for water flow in a glass-walled microchannel $\left(k_{1} / k_{2}=0.443\right)$. The heater size is $a / L=0.5$, the heater-sensor spacing is $b / a=1$, and the wall thickness is $W / L=0.5$.

The numerical simulation was run multiple times to explore the effects of sensor spacing $b / a$ and different wall materials. For each sensor geometry, the simulation was carried out for a series of $P e$ values so that the Peclet location of the amplitude peak in the temperature-difference signal could be identified. About 2400 simulations were carried out to obtain the information displayed in Figure 7, which shows the Peclet location of the peak amplitude versus frequency for $b / a=0.1,0.5,1.0$ and for water flow through channel walls of plastic, glass, and stainless steel (see Table 1 for thermal properties used here). As indicated earlier, this Peclet value represents the maximum measureable flow speed. Figure 7 shows that the wall material has a strong effect, with a higher-conductivity steel having a larger Peclet range than lower-conductivity plastic. The values for plastic are very close to fluid-only behavior (not shown). Sensor spacing $b / a$ has a smaller effect, with a similar trend for every wall material. The curves for glass and stainless steel do not extend to frequency $\omega^{+}=40$ because the simulation takes more computer time for larger Peclet numbers, and it was judged that small Peclet numbers are more important. The values shown, however, are sufficient to clearly define the trends. Variations in wall thickness were also explored in the range $0.1<W / L<1$, but there were only tiny differences in the maximum measurable Peclet. As long as the wall is thick, which is common for microchannels, the water-flow results are not sensitive to wall thickness. Gas flows were not included in this study.

The information given in Figure 7 may be used for sensor design for measurements in water flows, as follows. When the fluid velocity is large, high-conductivity walls, high frequencies, and smaller sensor spacing are appropriate for thermal measurements. For measurement of very small velocities, low conductivity walls, low-frequency heating, and larger sensor spacing is appropriate. Given a microchannel with a particular wall material, channel height, and flow range to be measured, the heating frequency and/or the sensor spacing can be chosen appropriately from Figure 7. In this way the sensor saturation, noted by experimental investigators, can be avoided.

The information displayed in Figure 7 was also used to construct a design correlation. The maximum Peclet value that can be observed with thermal sensing is given by: 


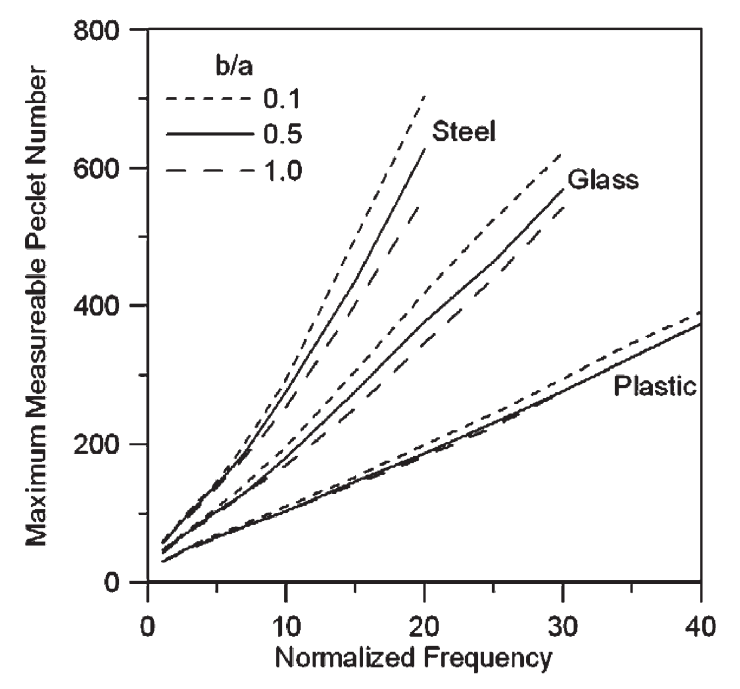

Figure 7. Maximum measurable Peclet number attainable in a thermal sensor in water flow over a range of frequencies, for three wall materials, and for three values of sensor spacing $b / a$. Fixed values are wall thickness at $W / L=0.5$ and channel size at $a / L=0.5$.

$$
\begin{gathered}
P e_{\max }=9.2\left(\omega^{+}\right)^{N}+195\left(k_{1} / k_{2}\right)^{-0.047}-165 \\
\text { where } \quad N=(b / a)^{-0.02} \cdot\left(\alpha_{1} / \alpha_{2}\right)^{-0.095}
\end{gathered}
$$

This relationship agrees with the Figure 7 data within $8.7 \%$ for plastic walls and within $4 \%$ for stainless steel walls for frequencies $\omega^{+}>3$. Some physical intuition about actual frequencies and flow velocities associated with dimensionless quantities $\omega^{+}$and $P e$ may be gained from values given in Table 2 . For water flow in smaller microchannels, Table 2 shows that it is the smaller frequencies and smaller flow rates that are important.

\section{Numerical issues}

The numerical calculations carried out in this research presented some challenges. The laminar fluid-flow GF is in the form of a series that converges slowly for large values of the Fourier-transform parameter $\beta$. Fortunately, the fluid-flow GF for large $\beta$ asymptotically approaches the shear-flow GF, available in non-series form [20], which was substituted at large $\beta$ values. This substitution provided a considerable improvement in computer time. Numerical quadrature for the inverse Fourier transform was carried out with an iterative Romberg scheme. The calculations were coded in Fortran 77 with variables of the type double-precision complex and compiled on a Sun unix workstation with dual $900 \mathrm{MHz}$ processors running the Solaris operating system. As an indication of the amount of computer time needed for the calculations presented in this paper, the $\omega^{+}=15$ curve from Figure 6 contains 60 points. The first 30

Table 2. Frequency and velocity values associated with dimensionless quantities $\omega^{+}$and $P e$ for water flow in microchannels.

\begin{tabular}{lllll}
\hline$L(\mathrm{~mm})$ & $\omega^{+}$ & $f(\mathrm{~Hz})$ & $P e$ & $U(\mathrm{~cm} / \mathrm{s})$ \\
\hline 0.5 & 1 & 0.9 & 1 & 0.29 \\
0.5 & 10 & 9.4 & 10 & 2.9 \\
0.5 & 100 & 94.0 & 100 & 29.0 \\
0.1 & 1 & 23.0 & 1 & 1.47 \\
0.1 & 10 & 230.0 & 10 & 14.7 \\
0.1 & 100 & 2300.0 & 100 & 147.0 \\
\hline
\end{tabular}

points on $(0.1<P e<10)$ were computed in $48 \mathrm{~s}$ of computer time, and the last 30 points on $(10<P e<1000)$ were computed in about $30 \mathrm{~min}$ (the hypergeometric series converges more slowly at larger Pe). Because of the relatively small computer time for evaluating these theory-based results, it has been possible to simulate thousands of different cases as part of this project. This level of exploration would not have been practical with a finite-difference or finite-element method.

\section{Conclusion}

In this paper theory for the temperature is given for laminar flow in a parallel-plate microchannel caused by steadyperiodic heating in the channel wall. The temperature expressions have the form of integrals, developed by the method of GF, and numerical results have been computed by quadrature. The fluid-side GF has the form of a hypergeometric series that has been checked by comparison with shear-flow at high flow rates and high frequencies, and it has been checked against heat conduction solutions at low flow rates.

The application is flow measurement in microchannels by thermal methods. A variety of results have been presented for the temperature on the channel wall, from which the following conclusions are drawn. First, as flow increases, the temperature amplitude on the heater first increases to a peak value and then decreases. This trend, observed experimentally by others, is caused by a progression from heat transfer dominated by axial conduction upstream, to heat transfer involving both axial conduction and downstream convection, and finally to heat transfer dominated by downstream convection. Second, this temperature peak is identified as the maximum flow value that can be observed by a thermal measurement involving a heater alone, or a heater and two sensors. This maximum observable flow value can be adjusted somewhat by changing the heating frequency. Third, because the theory-based expressions can be evaluated relatively rapidly, a great many cases have been computed to simulate the sensor under a variety of flow speeds, heating frequencies, and sensor geometries. This information is presented in the form of a design correlation for matching a particular sensor to its flow-measurement range.

The solution method presented here could be extended to other sensor geometries and other flow situations by developing the appropriate Green's functions. For example, this method could be applied to microchannels with layered walls, to heating distributed throughout the channel wall, to other velocity distributions that arise in microchannel flows, and to flow in circular tubes.

Acknowledgments - The author would like to acknowledge funding from the University of Nebraska Foundation under the Layman Program. Thanks also to undergraduate students Eric Wood and Jared Miller who meticulously ran computer codes and plotted results.

\section{References}

[1] M. L. Michelsen and J. Villadsen, The Graetz problem with axial heat conduction, Int. J. Heat Mass Transfer 17 (1974), pp. 1391-1402.

[2] Y. Bayazitoglu and M. N. Ozisik, On the solution of Graetz type problems with axial conduction, Int. J. Heat Mass Transfer 23 (1980), pp. 1399-1402.

[3] T. V. Nguyen, Laminar heat transfer for thermally developing flow in ducts, Int. J. Heat Mass Transfer 35 (7) (1991), pp. 1733-1741. 
[4] A. Haji-Sheikh, Determination of heat transfer in ducts with axial conduction by variational calculus, J. Heat Transfer 131 (9) (2009), pp. 091702.1-091702.11.

[5] I. Tiselj, G. Hetsroni, and B. Mavko et al., Effect of axial conduction on heat transfer in micro-channels, Int. J. Heat Mass Transfer 47 (2004), pp. 2551-2565.

[6] A. Haji-Sheikh, J. V. Beck, and D. E. Amos, Axial heat conduction effects in the entrance region of parallel-plate ducts, Int. J. Heat Mass Transfer 51 (2008), pp. 5811-5822.

[7] G. Marananza, I. Perry, and D. Maillet, Mini-and micro-channels: influence of axial conduction in the walls, Int. J. Heat Mass Transfer 47 (2004), pp. 3993-4004.

[8] B. Weigand and D. Lauffer, The extended Graetz problem with piecewise constant wall temperature for pipe and channel flows, Int. J. Heat Mass Transfer 47 (2004), pp. 5303-5312.

[9] B. Weigand and G. Gassner, The effect of wall conduction for the extended Graetz problem for laminar and turbulent channel flows, Int. J. Heat Mass Transfer 50 (2007), pp. 1097-1105.

[10] J. Lahjomri, A. Oubarra, and A. Alemany, Heat transfer by laminar Hartmann flow in thermal entrance region with a step change in wall temperatures: the Graetz problem extended, Int. J. Heat Mass Transfer 45 (2002), pp. 1127-1148.

[11] K. Zniber, A. Oubarra, and J. Lahjomri, Analytical solution to the problem of heat transfer in an MHD flow inside a channel with prescribed sinusoidal wall heat flux, Energy Conver. Manage. 46 (2005), pp. 1147-1163.

[12] W. J. Minkowycz and A. Haji-Sheikh, Heat transfer in parallel plates and circular porous passages with axial conduction, Int. J. Heat Mass Transfer 49 (2006), pp. 2381-2390.

[13] B. Cetin, A. G. Yazicioglu, and S. Kakac, Fluid flow in microtubes with axial conduction including rarefaction and viscous dissipation, Int. Comm. Heat Mass Transfer 35 (5) (2008), pp. 535-544.

[14] B. Cetin, A. G. Yazicioglu, and S. Kakac, Slip-flow heat transfer in microtubes with axial conduction and viscous dissipation - an extended Graetz problem, Int. J. Therm. Sci. 48 (2009), pp. 1673-1678.
[15] M. Unsal, A solution for the complex eigenvalues and eigenfunction periodic Graetz problem, Int. Comm. Heat Mass Transfer 25 (4) (1998), pp. 585-592.

[16] B. Fourcher and K. Mansouri, An approximate analytical solution to the Graetz problem with periodic inlet temperature, Int. J. Heat Fluid Flow 188 (2) (1997), pp. 229-235.

[17] G. Bedo, H. Fannasch, and R. Muller, A silicon flow sensor for gases and liquids using AC measurements, Sensors Actuators 85 (2000), pp. 124-132.

[18] W. P. Yan, C. Liu, and J. Li et al., Thermal distribution microfluidic sensor based on silicon, Sensors Actuators B 108 (2005), pp. 943-946.

[19] A. Mandelis, Diffusion-Wave Fields, Mathematical Methods and Green's Functions, Springer, New York (2001).

[20] K. D. Cole, Flush-mounted steady-periodic heated film with application to shear-stress measurement, J. Heat Transfer 130 (11) (2008), pp. 111601.1-111601.10.

[21] J. V. Beck, K. D. Cole, A. Haji-Sheikh, and B. Litkouhi, Heat Conduction Using Green's Functions, Hemisphere, New York (1992).

[22] W. A. McGahan and K. D. Cole, Solution of the heat conduction equation in multilayers for photothermal deflection experiments, J. Appl. Phys. 72 (1992), pp. 1362-1373.

[23] C. H. Park and K. D. Cole, Unsteady heat transfer from a thickfilm sensor, AIAA J. Thermophys. Heat Transfer 8 (4) (1994), pp. 797-799.

[24] D. G. Cahill, Thermal conductivity measurement from 30 to $750 \mathrm{~K}$ : the $3 \omega$ method, Rev. Sci. Instrum. 61 (1990), pp. 802-808.

[25] Y. A. Melnikov, Influence Functions and Matrices, Marcel Dekker, New York (1999).

[26] K. D. Cole, Steady-periodic Green's functions and thermal-measurement applications in rectangular coordinates, J. Heat Transfer 128 (7) (2006), pp. 709-716. 